\title{
Protein aggregation in disease: a role for folding intermediates forming specific multimeric interactions
}

\author{
Arthur Horwich \\ Howard Hughes Medical Institute and Department Of Genetics, Yale University School Of Medicine, New Haven, Connecticut, USA \\ J. Clin. Invest. 110:1221-1232 (2002). doi:10.1172/JCI200216781.
}

For many decades, clinicians have been aware of the formation of insoluble protein aggregates in particular diseases. For example, in sickle cell disease, the polymerization of sickle hemoglobin into fibrils in the deoxygenating environment of the microvasculature causes sickling of red cells and vaso-occlusion (1). In the most common form of $\alpha_{1}$-antitrypsin deficiency (see Perlmutter, this Perspective series, ref. 2), the presence of $\alpha_{1}$-antitrypsin inclusions in the endoplasmic reticulum (ER) of hepatocytes is associated with deficient release of the enzyme into the circulation, placing both the inclusion-laden liver and the antitrypsin-deficient lung at risk for damage (3). In Alzheimer disease (see Selkoe, this series, ref. 4), the presence in the CNS of $\beta$-amyloid-containing plaques is associated with neurodegeneration and dementia (5). Similarly, other neurodegenerative diseases have recently been discovered to involve protein aggregation. For example, prion diseases such as Creutzfeldt-Jacob disease and bovine spongiform encephalopathy are associated with amyloid deposits of the PrP protein (6). Polyglutamine repeat diseases such as Huntington disease are likewise associated with neuronal cytosolic and intranuclear inclusions (7). These inclusions are composed of fibrils that stain similarly to amyloid (8). Finally, in Parkinson disease, inclusions known as Lewy bodies, found in the cytoplasm of cells of the basal ganglia, include amyloidlike aggregates of the protein $\alpha$-synuclein $(9,10)$.

What do we know about the various processes that produce these aggregates? Are there cellular mechanisms that can either prevent or respond to such processes? Can current understanding translate into prevention or therapy? The various articles in this Perspective series address these questions and demonstrate the considerable progress that has been made toward explaining these events in molecular terms.

\footnotetext{
Address correspondence to: Arthur Horwich, Howard Hughes Medical Institute and Department of Genetics, Yale University School of Medicine, Boyer Center, 295 Congress Avenue,

New Haven, Connecticut 06510, USA.

Phone: (203) 737-4431; Fax: (203) 737-1761;

E-mail: horwich@csb.yale.edu.

Conflict of interest: No conflict of interest has been declared.

Nonstandard abbreviations used: endoplasmic reticulum (ER); phosphoglycerate kinase (PGK); bovine growth hormone (bGH); transthyretin (TTR).
}

Resting on such understanding are new therapeutic strategies for some of these diseases, several of which are being tested in animal model systems.

Here, I offer an overview of the molecular mechanisms of both protein folding and misfolding, particularly in the formation of aggregates. This Perspective first revisits some of the seminal developments in protein biochemistry that led to the idea that protein aggregates contain specific, organized, polymeric structures formed from partly structured folding intermediates by alternative, off-pathway folding steps. The focus then turns to cellular responses in which aggregation is prevented or reversed by molecular chaperones, molecules dedicated to providing kinetic assistance to protein folding. The final section focuses on the structure and mechanism of formation of amyloid aggregates and comments on the prospects for treating the diseases with which their formation is associated.

\section{Folding pathways of small and large proteins: studies in vitro and in bacteria}

Two-state folding of small proteins. Mechanistic studies of both productive protein folding and misfolding/aggregation have been considerably advanced by the ability to observe these reactions in vitro with purified proteins. A purified native protein can be unfolded using denaturants such as $6 \mathrm{M}$ guanidine- $\mathrm{HCl}$ or $8 \mathrm{M}$ urea, then diluted from denaturant into aqueous buffer and allowed to fold or misfold. In the case of an enzyme, proper folding to native form can be detected by acquisition of enzymatic activity; for other proteins, the acquisition of physical properties characteristic of the native state must be monitored directly. The first such experiment was carried out by Christian Anfinsen and his coworkers in the late 1950s on the enzyme ribonuclease A (11). The ability of the reduced and unfolded protein to spontaneously fold into its native state established that the primary amino acid sequence of a protein contains all of the information necessary for proper folding into native form, a fundamental principle for which Anfinsen received the Nobel Prize in Chemistry in 1972. The studies of Anfinsen and colleagues also implied that the native state of a protein lies at a free energy minimum and indicated a thermodynamic contribution to the folding process.

A large number of proteins of fewer than 150 amino acids can efficiently refold upon dilution from denat- 

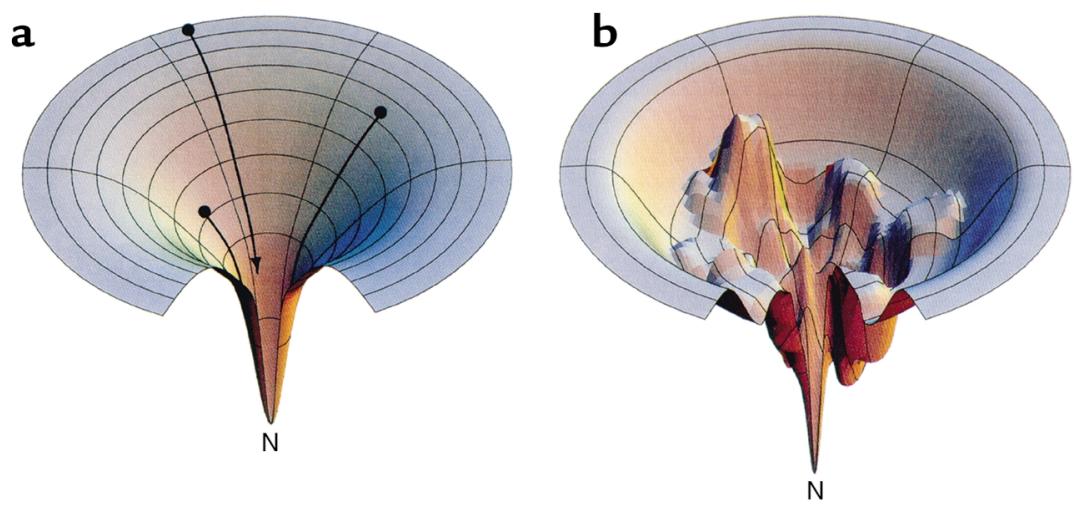

Figure 1

Funnels representing an idealized energy landscape for protein folding (a) or a rugged energy landscape with kinetic traps (b), from models of Dill and Chan (80). (a) The unfolded chain starts at the top of an energy landscape, and, as it forms intrachain contacts, lowering its free energy (and descending the funnel), the number of conformations it can sample is progressively reduced, thus eliminating the need for a global search. Ultimately, it reaches the unique native state at the energetic minimum. Note that the number of pathways typically available coming down from the unfolded state may not be unlimited, as depicted here, but may be multiple. (b) Descending a rugged landscape, the polypeptide can lodge in a kinetic trap or can alternatively descend through a narrow path to the native state. Molecular chaperones may act to "smooth" rugged landscapes, preventing polypeptide from descending into a kinetic trap, or rescuing it from one. Reproduced with permission from Nature Structural Biology (80). N, native state.

Partly folded intermediate states in the folding and aggregation of larger proteins. In contrast to the two-state proteins, refolding from denaturant of many proteins of more than 100-150 amino acids does not proceed efficiently. Indeed, in many such experiments, a precipitate of aggregated protein is recovered at the bottom of the tube. This finding implies that for many larger proteins, as well as for some smaller ones (see refs. 14, 15 for examples), there are kinetic barriers to reaching the native state. Thus, when left to fold on their own, these proteins become "kinetically trapped" in local energetic minima (Figure 1b). It is such intermediate, partly folded conformations that can associate to form aggregates. Some such intermediate states may be obligatory ones, whose conformation a folding protein must necessarily traverse in order to reach native form, whereas others may lie away from a productive pathway. Regardless, when such states are sig-

urant, many of them exhibiting what are called twostate kinetics, that is, traversing only between the unfolded and native states, usually on the time scale of a few seconds or less. A wealth of studies of such proteins has begun to define the modes by which proteins fold into their native forms. The process does not appear to be random, as the search time required would be impossibly long; rather, the unfolded protein undergoes specific kinetically preferred steps on the way to the native state, albeit that there may be multiple choices at any given point (see Figure 1a). The ability to examine the transition states for two-state folding reactions by mutation analyses, pioneered by Alan Fersht and his colleagues, allows determination of the elements of structure that are present at the transition state and provides information on which structural features of the native protein have formed during folding up to a given point (12). Proteins appear to follow multiple approaches to the transition state. For example, some proteins rapidly acquire secondary structure (which is present already at the transition state) before organizing tertiary structure; for others, a rapid collapse of hydrophobic regions to form a core can occur before or at the same time as secondary and tertiary structure formation. An interesting observation from the recent work of David Baker and coworkers is that proteins whose contiguous amino acid sequences remain in contact with each other via secondary or tertiary structure in the final three-dimensional native state fold faster than those whose local structures are formed from distantly separated sequences (in a parallel $\beta$-sheet, for instance), implying an entropic penalty for bringing together the distant segments of polypeptide in the latter proteins (13). nificantly populated, intermediate species are able to interact specifically with each other to form aggregates.

Early evidence for this model of aggregate formation came from the classic experiment of Michel Goldberg and coworkers, who examined the behavior of the tetrameric enzyme tryptophanase, diluted from urea (16). Goldberg and colleagues observed that, after first unfolding tryptophanase with $8 \mathrm{M}$ urea and then diluting into buffer, both refolded native enzyme and inactive aggregate formed. With increasing concentrations of the protein, however, the yield of active enzyme was reduced, and the inactive enzyme sedimented in a sucrose gradient as a polydisperse population of molecules. Importantly, these workers showed that the protein partitions in different ways between the native state and the inactive aggregate, depending on the concentration of urea used at the renaturation step. In particular, at $3 \mathrm{M}$ urea, all of the protein is recovered in the aggregate, and no recovery ensues following removal of urea, suggesting that a folding intermediate is involved whose population is favored at this concentration of urea. Goldberg and his associates further showed that the self-association of this intermediate is specific, since when BSA or even a crude bacterial extract is denatured in the same tube with the tryptophanase, the enzyme's refolding proceeds exactly as before. Hence, for any given concentration of tryptophanase, contacts between tryptophanase monomers, leading to either productive or nonproductive folding, are not affected by the presence of other denatured proteins. To account for both the concentration dependence and the specificity of the reaction, Goldberg and colleagues proposed that aggregation is driven by the same stereospecific interactions that are required for folding of 
the polypeptide chain to the native form, but that these interactions occur between two separate chains (Figure 2); that is, intermolecular contacts become substituted for intramolecular ones.

Soon after, Rainer Jaenicke and his coworkers performed a study of refolding and aggregation of porcine tetrameric lactate dehydrogenase that revealed a similar protein concentration-dependent behavior of aggregation (17). Using far ultraviolet circular dichroism spectroscopy, these authors showed that the aggregates resemble the native protein with regard to their $\alpha$-helical and $\beta$-strand content, providing direct support for the idea that monomeric intermediate states responsible for aggregation exhibit significant nativelike secondary structure. Unlike the rapid, first-order reaction for productive folding, the kinetics observed for aggregate formation was consistent with a competition between two types of events, a unimolecular, concentration-independent intrachain interaction, and a multimolecular, interchain one whose rate increases rapidly with the protein concentration.

Secondary structures and hydrophobic interactions that drive protein aggregation. More recent studies of the in vitro refolding of two monomeric proteins, phosphoglycerate kinase (PGK) and bovine growth hormone (bGH), provide information on the structures mediating aggregating interactions. In the case of PGK, the kinetics of aggregate formation is biphasic, with a first phase exhibiting first-order kinetics, corresponding to folding of an intermediate, and a second phase that is concentration-dependent. This second stage can be suppressed by chilling to $4^{\circ} \mathrm{C}$, consistent with involvement of hydrophobic interactions in producing contact between the monomer intermediates (18). The aggregated species lacks substantial $\alpha$-helical structure, normally present at the surface of native PGK, and apparently exposes hydrophobic core $\beta$-strands. Presumably, some stereospecific combination of hydrophobic and $\beta$-strand interactions between monomers constitutes the interchain interactions of aggregating PGK.

In experiments with bGH, as in the other studies, the intermediate species that accumulates shows considerable native-like secondary structure (in this case all $\alpha$-helical), but it lacks the native tertiary structure. The unfolded bGH partitions between the aggregated and the native states (19). Remarkably, addition of a specific peptide fragment (amino acids 96-133) of bGH can block aggregation. This peptide has been proposed to form an amphiphilic $\alpha$-helix that associates through its hydrophobic aspect with a homologous exposed hydrophobic aspect of the same $\alpha$-helix in the intermediate form of bGH, thus preventing intermolecular interaction and consequent aggregation.

Inclusion body formation in vivo. Elegant studies of Jonathan King and coworkers, examining two Salmonella phage proteins, show that the kinetic partitioning mechanism observed in vitro operates in vivo as well. These authors have studied the $\mathrm{P} 22$ tailspike protein, a homotrimeric assembly of 666 amino acid subunits that comprises a coiled array of parallel $\beta$-strands taking the shape of a fish (ref. 20; subunit shown in Figure 3a), as well as a $48-\mathrm{kDa}$ coat protein from the same bacteriophage. King and colleagues have used temperature-sensitive mutations in the tailspike protein to dissect the pathway of folding and assembly in intact Salmonella (21) (Figure 3b). At high temperature, the newly synthesized tailspike subunits are initially found in a soluble fraction, but they then aggregate, forming refractile, dense, particulate structures known as inclusion bodies, which sediment at low speeds after cell breakage. By contrast, at lower (permissive) temperature, the newly made tailspike subunits remain soluble, assembling into the native homotrimer. When cells incubated at high temperature are downshifted within a few minutes of synthesis, the tailspike subunits that were initially soluble can be recovered in the homotrimeric form, the result of redirecting the partitioning of an apparent thermolabile folding intermediate toward the native state. However, if downshift is carried out at later times, subunits cannot be recovered into the soluble fraction or into a native trimer; aggregation and inclusion body formation supervene and become irreversible (Figure 3b).

King and coworkers suggested that their temperature-sensitive folding mutants, which map broadly across the middle 200 amino acids of the tailspike, in the midsection of the "fish" (Figure 3a, blue spheres), promote the conformational change of a thermolabile folding intermediate (designated I in Figure $3 b$ ) to yield aggregates. This intermediate, which exists in equilibrium with the other conformational species even in the wild-type setting, forms a species that can self-associate (22). The crystal structure of the tailspike tends to support this model (20), as the mutations map to the $\beta$-coil domain of the native structure (Figure 3a), mostly to solvent-exposed positions or to residues at or near the tight $\beta$-turns that enable formation of the $\beta$-coil. As such, these mutations would not be expected to affect the native structure, but they would be critical during
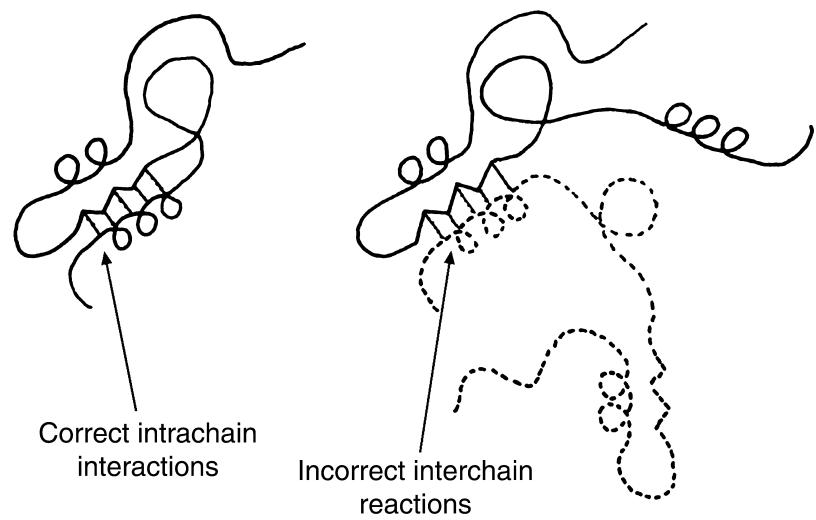

\section{Figure 2}

Model of Goldberg and coworkers for protein aggregation involving specific structural contacts formed between two polypeptide chains instead of within a single chain (16). The same bonds are formed but promote incorrect quaternary associations. Kinetic competition between these interchain and intrachain contacts is concentration-dependent, and such contacts are formed from intermediate states (see text). Reproduced with permission from European Journal of Biochemistry (16). 
a
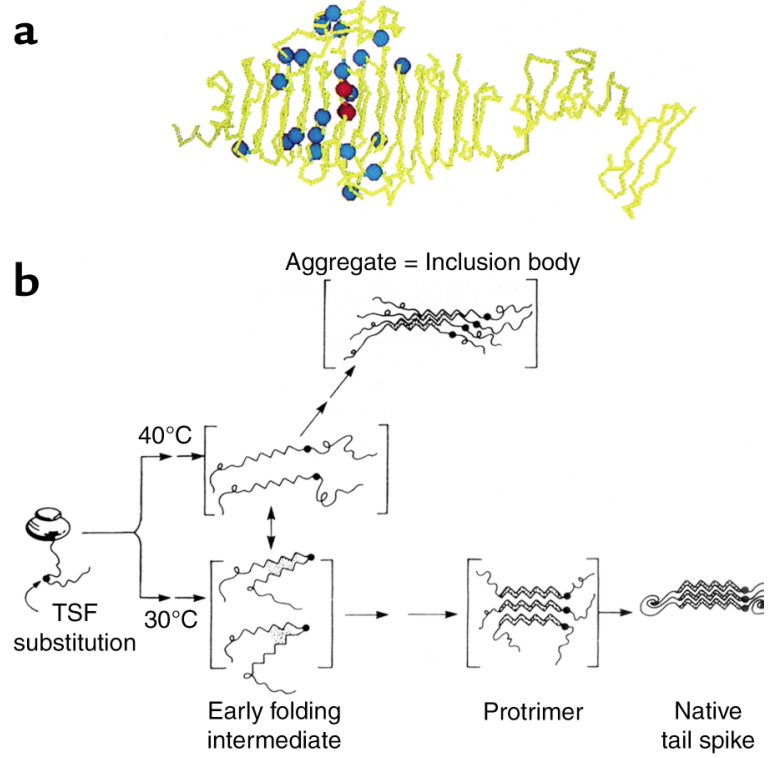

(I)

C

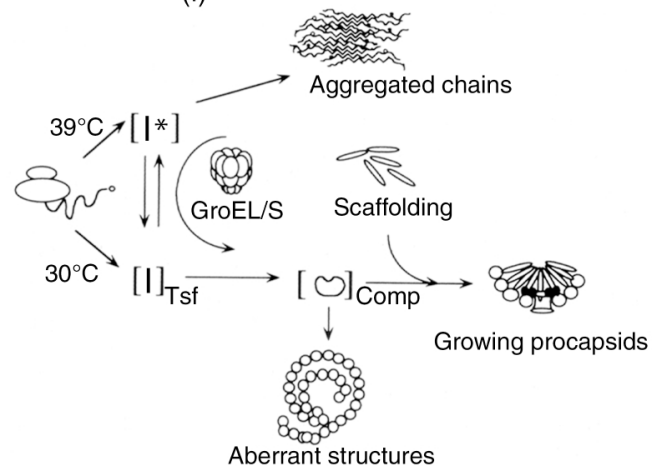

folding for producing the proper coiling and registering of the $\beta$-coil (Figure $3 b$ ).

Remarkably, suppression mutagenesis identifies two specific second-site mutations that can block the aggregation phenotype of the temperature-sensitive tailspike mutants (23). These global suppressor mutations, mapping into the same central region of the tailspike (Figure 3a, red spheres), are only active when present in the same subunit as the original mutation and do not affect the stability of the native (double-mutant) protein. They therefore appear to favor partitioning of the monomeric tailspike folding intermediate to native trimeric form (Figure 3b), either by stabilizing the on-pathway intermediate forms or by destabilizing off-pathway conformations that lie in the direction of aggregation.

Temperature-sensitive folding mutations in the P22 coat protein behave similarly to the tailspike with respect to temperature-dependent partitioning into aggregates in vivo (24) (Figure 3c). King and colleagues have observed the folding and aggregation pathways of both tailspike and coat using nondenaturing gel analysis, an in vitro procedure that allows species from monomer up to higher-order multimers of either protein to be observed directly. Very interestingly, the two proteins, when placed together under conditions favoring aggregation, produce two distinct ladders of multimers in the
Figure 3

Protein misfolding and inclusion body formation studied in vivo with the phage P22 system. (a) Crystal structure of a subunit of the P22 tailspike homotrimer (20), showing the parallel $\beta$-coil structure and ventral and dorsal "fins." Blue spheres represent positions of temperature-sensitive folding mutants (21), which in general occupy solvent-exposed sites in this native form (see text). Two red spheres represent two global suppressor mutations (23). Reproduced with permission from The FASEB Journal (22) (b) Pathways of folding and aggregation in vivo of the phage P22 tailspike protein bearing temperature-sensitive folding mutations. The single-chain folding intermediates are thermolabile (designated I), with the mutations, potentially affecting turn formation, favoring off-pathway intermediate formation and association over productive folding. The global suppressor mutations reverse such partitioning, favoring the productive early folding intermediate over the aggregation-producing one (vertical arrow), even at $40^{\circ} \mathrm{C}$. Reproduced with permission from Science (23). TSF, temperaturesensitive folding. (c) Folding and aggregation in vivo of phage P22 capsid protein bearing temperature-sensitive folding mutations (24). Pathways are similar to those for the tailspike. Here, the chaperonin system, GroEL/GroES/ATP, can act at $39^{\circ} \mathrm{C}$ to bind the aggregation-prone I* intermediate (monomer) and facilitate its productive folding. Reproduced with permission from The Journal of Biological Chemistry (24).

native gel, one corresponding to that observed with tailspike aggregating alone and the other corresponding to that observed when coat protein aggregates alone (25). This experiment provides the clearest evidence that proteins aggregate independently of one another and strongly suggests that specific contacts, formed between folding intermediates, produce aggregates that are ordered arrays of a single protein.

Domain swapping between monomers. Surprising findings from David Eisenberg's group, comparing the crystal structure of diphtheria toxin as a native monomer and as an acid-induced dimer, also have important implications for aggregation processes in general (26). These authors observed in the dimeric form that one of the three domains of the toxin monomer, the receptor-binding domain, had become rearranged in position such that its noncovalent contacts with the two other domains had been broken; it had rotated $180^{\circ}$ and translated as much as $65 \AA$ to form normal noncovalent contacts with the two domains of the other chain of the dimer (Figure 4, a and b). Such domain swapping has now been observed in crystals of several dozen other proteins. In all cases, as with Goldberg and colleagues' early model of aggregation, the behavior is one of forming an intersubunit interface that is identical to an intrasubunit interface. In some cases, exchange observed in the crystals involves three or four subunits. The potential relevance of such higher-order exchange to processes of protein aggregation seems inescapable, as Eisenberg and coworkers consider the possibility of higher-order domain-swapped oligomers produced as closed or as linear assemblies (27) (Figure 4c). Indeed, the aggregation of ZZ-type $\alpha_{1}$-antitrypsin (see Lomas and Mahadeva, this Perspective series, ref. 28) appears to be just the latter type of arrangement, in that a reactive center loop segment, which normally inserts as a $\beta$-strand into a gap in the center of a sheet within the same molecule, 
inserts instead into the same position of another molecule, a process termed loop-sheet polymerization (29). More detailed structural studies of additional aggregates will be necessary to evaluate whether domain swapping operates more generally or whether aggregates can be formed by intersubunit contacts that have no equivalent in the native state.

\section{Protein folding in the cell: chaperones and protein biosynthesis}

Binding of folding intermediates by chaperones. Work of the 1980s and early 1990s established that a class of specialized heat-inducible proteins, molecular chaperones, can provide kinetic assistance to protein folding in a variety of contexts, in general preventing protein aggregation and promoting proper folding by binding to hydrophobic surfaces specifically exposed in non-native protein conformations $(30,31)$. Such behavior has been especially well demonstrated for the double-ring assemblies known as chaperonins. For example, a host of proteins imported into yeast mitochondria in vivo fail to reach their refolded native state and become insoluble in a mutant deficient in the mitochondrial chaperonin Hsp60 (32). In vitro, likewise, subunits of such proteins as Rubisco, rhodanese, and malate dehydrogenase undergo wholesale aggregation following dilution from denaturant, failing to recover enzymatic activity. But the presence of the bacterial chaperonin GroEL, which stoichiometrically binds proteins in non-native states (33-35), allows these enzymes to be recovered quantitatively in native form via a refolding reaction requiring the cochaperonin GroES and ATP. With structural analysis of GroEL, it became clear that its open rings provide a hydrophobic binding surface (Figure 5a) that recognizes exposed hydrophobic surfaces specific to non-native polypeptides, surfaces that become buried in the interior of native proteins (36). These same hydrophobic surfaces in non-native proteins contribute, as outlined above, to the process of multimolecular aggregation, albeit that particular secondary-structural elements appear also to be involved. Nevertheless, the cavity surface of GroEL successfully competes for these hydrophobic sites - apparently without regard to their structural context, since both all- $\alpha$-helical proteins and $\beta$-sheet-rich ones can be bound (reviewed in ref. 37). Binding through hydrophobic contacts prevents such surfaces from promoting aggregation.

Not only can GroEL prevent aggregation via the step of binding, but it can also actively promote productive folding, triggered upon binding of ATP and GroES to the same ring to which the substrate polypeptide binds $(31,38)$ (Figure 5, a and b). Such binding is associated with rigid body movements of the peptide-binding apical domains of the bound GroEL ring, elevating and twisting their hydrophobic surfaces away from the central cavity and replacing the lining of the cavity with hydrophilic surfaces. These movements result in rapid release of the substrate protein from the cavity walls into a now encapsulated chamber, where it has no access to other monomers with which it could aggregate. The walls of the cavity, now hydrophilic in character, may encourage productive folding, insofar as they energeti-

\section{Figure 4}

Domain swapping between protein monomers in crystals. ( $\mathbf{a}$ and $\mathbf{b}$ ) Original demonstration of domain swapping of diphtheria toxin in a protein crystal (26). The receptor-binding domain of a monomer (shown in yellow in a) can become rotated and translated to form contacts with the other two domains of the toxin, as in $\mathbf{b}$, where the blue receptor-binding domain of the subunit at the left has become associated with the other two domains of the green subunit at the right, and vice versa. This demonstrates the substitution of interchain contacts for intrachain ones as proposed by Goldberg (see Figure 2). R, receptor binding domain. Reproduced with permission from Proceedings of the National Academy of Sciences of the United States of America (26). (c) Models for mechanics of higher-order domain swapping. Reproduced with permission from Protein Science (27). a
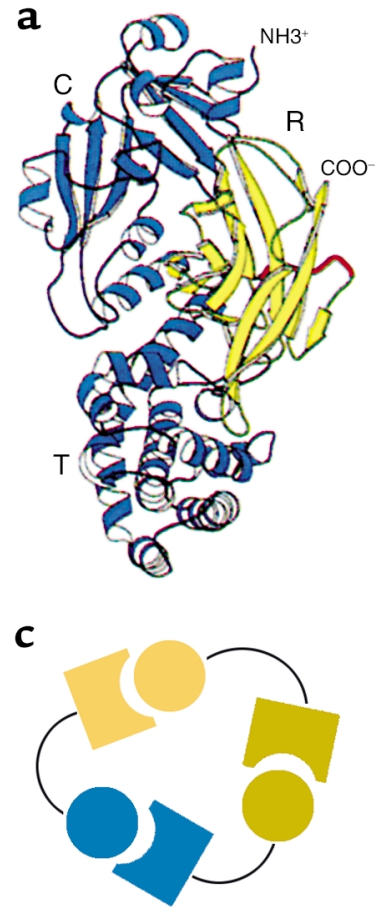

Cyclic (closed) domain-swapped oligomer
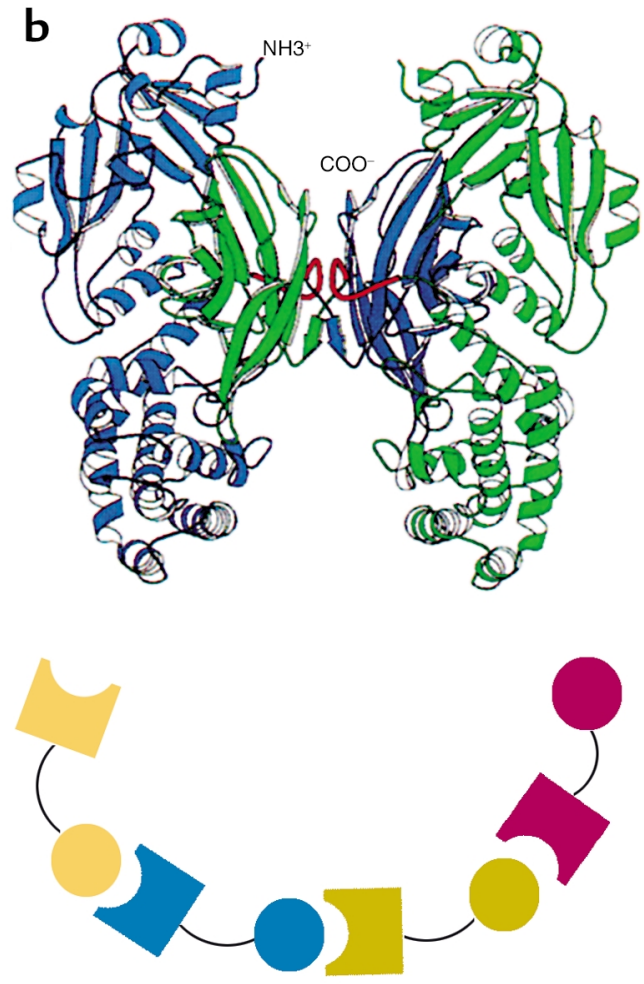

Linear (open-ended) domain-swapped oligomer 

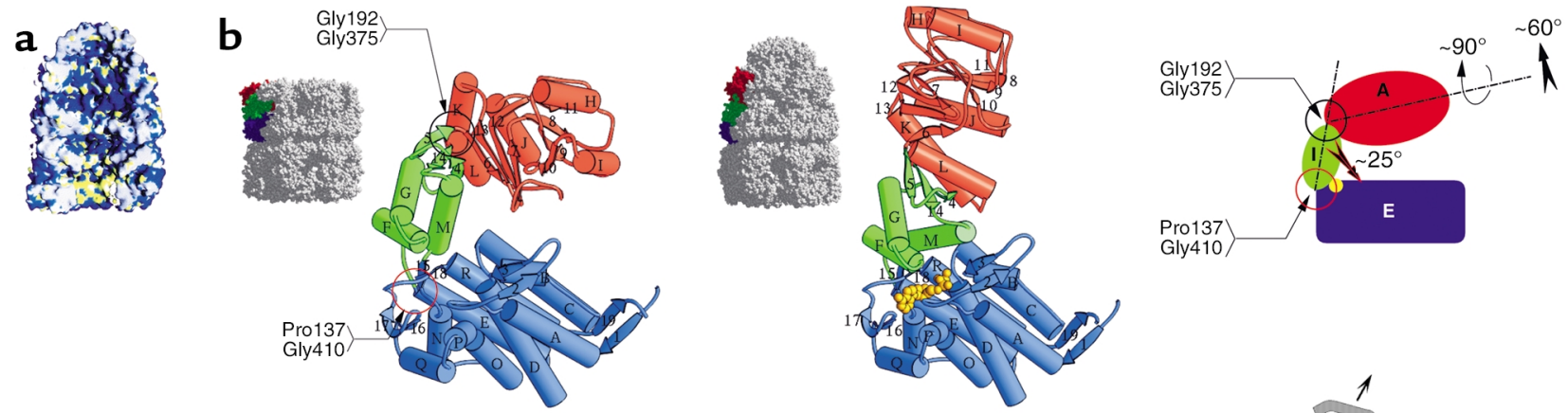

c
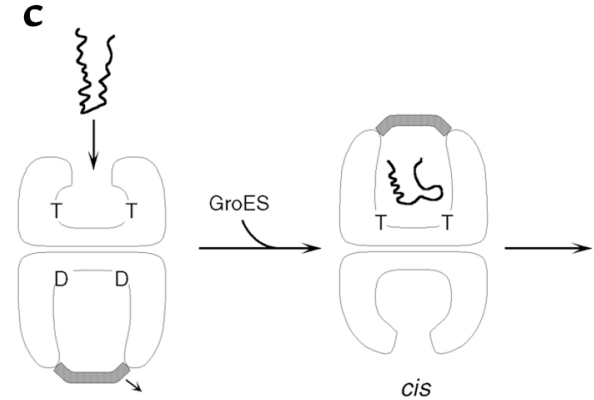

Polypeptide binding

Folding triggered

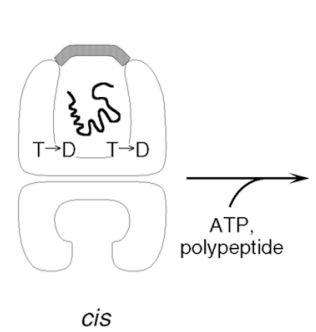

ES release primed

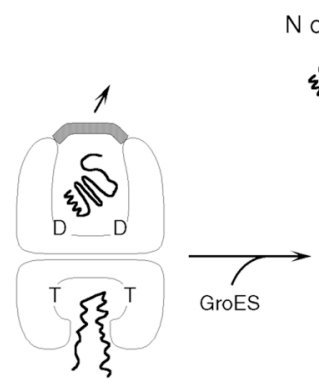

Discharge triggered

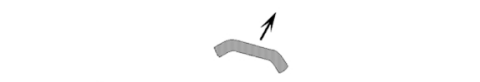

Figure 5

Chaperonin-mediated folding by GroEL-GroES. (a) Space-filling model of an asymmetric GroEL/GroES chaperonin complex showing GroES bound as a dome-shaped molecule to the upper GroEL ring with the lower GroEL ring open. The cavity of the lower ring displays a hydrophobic surface (yellow) that can accept non-native polypeptides exposing hydrophobic surface, while the cavity of the upper GroES-bound ring has displaced the hydrophobic surfaces away from the cavity, substituting them with hydrophilic surface (blue) that may serve to favor folding of the encapsulated polypeptide within this cavity. (b) Rigid body movements, associated with GroES binding to a GroEL ring, entail the elevation and twisting of the GroES-bound GroEL apical domains. This conformational change removes the hydrophobic surface from the cavity and replaces it with hydrophilic surface. Reproduced with permission from Nature (38). (c) Action of ATP binding and hydrolysis to advance the chaperonin cycle. ATP (designated as T; ADP as D) binds with positive cooperativity within one GroEL ring, but with negative cooperativity between rings, so that ATP effectively occupies only one ring at a time. This establishes the asymmetry of the system, which is reinforced by the nucleotide requirement for GroES binding. Nonnative polypeptide binds to the open ring of an asymmetric complex (first panel), and GroES binding to the same ring forms a folding-active complex and triggers polypeptide folding (second panel). The lifetime of this complex is determined by ATP hydrolysis in the GroES-bound ring, which weakens the interaction between GroES and GroEL (third panel). Binding of ATP and polypeptide to the opposite ring (fourth panel) then discharges GroES and the polypeptide, either in the native state $(\mathrm{N})$ or one committed to it $\left(\mathrm{I}_{\mathrm{c}}\right)$ or in a still non-native state $\left(\mathrm{I}_{\mathrm{uc}}\right)$ that can rebind to GroEL and try again to refold (fifth panel). Binding to an open GroEL ring may be associated with an unfolding action. Note that the rings oscillate back and forth as polypeptide-accepting and then folding-active, a function of the asymmetric binding of ATP/GroES. cis, binding of GroES and polypeptide to the same GroEL ring. Reproduced with permission from Current Opinion in Structural Biology (37).

cally favor conformations in which the substrate's hydrophobic surfaces are buried and its hydrophilic surfaces are exposed, as occurs in native proteins.

This folding-active ternary complex has a finite lifetime $\left(12\right.$ seconds at $23^{\circ} \mathrm{C}$ ) before the hydrolysis of ATP in the proximal ring and the subsequent binding of ATP to the opposite ring cause it to dissociate, releasing both GroES and the polypeptide substrate (31) (Figure 5c). If the protein has reached native form during this time, it proceeds to carry out its function; in the case of oligomeric proteins, assembly with other subunits occurs in the bulk solvent. If the protein has failed to reach native form, or one committed to completing folding to the native state, it becomes rebound by the chaperonin or by another chaperone in the same cellular compartment, and it can attempt once again to fold correctly. Thus, chaperonins play into the kinetic partitioning schemes outlined earlier for aggregation processes, shunting those folding intermediates that they recognize away from multimolecular aggregation and toward the native state. An excel- lent case of such partitioning is found in the P22 system, where aggregation of folding-defective mutant coat proteins has been observed in vivo to be suppressed by overexpression of GroEL and GroES (39) (Figure 3c).

Other cellular chaperones that reverse or prevent aggregation. Once aggregation supervenes, chaperonins such as GroEL are unable to reverse the process, probably because they can only bind free monomeric subunits. In contrast, another ring-shaped cylinder, Hsp104, appears able to mediate ATP-dependent disaggregation of "soft" aggregates of up to $600 \mathrm{kDa}$; the action of additional chaperones of the Hsp70 family allows the components of such aggregates, once released, to refold and attain the native conformation (40). In vivo in intact yeast, such action is accomplished in the cytosol, where the heat-inducible Hsp 104 protein can dissolve morphologic aggregates produced during heat shock following temperature downshift (41).

In addition to the active role played by chaperonins in productive folding, a number of other chaperones 
bias kinetic partitioning in vivo away from aggregation. These include Hsp70s and their cooperating DnaJrelated partners, Hsp40s, which recognize and bind exposed hydrophobic side chains of non-native proteins in the context of an extended conformation (31). Similarly, small heat shock proteins (sHsp's) of about $20 \mathrm{kDa}$ prevent aggregation by forming oligomeric assemblies that bind incipiently denaturing proteins at their surfaces during heat shock or other cellular stress. In the case of Hsp70 proteins, ATP binding drives release of bound protein, allowing it a chance to fold correctly. In contrast, a return to normal cellular conditions is associated with release from sHsp's.

A role of Hsp70 proteins in conformational quality control is highlighted by the ER version, BiP, which is involved with translocation of importing polypeptides and with their folding and assembly. Notably, BiP remains bound to many misfolded, incompletely modified, or incompletely assembled species in this cellular compartment, preventing exit and progression down the secretory pathway. In many cases, misfolded proteins are retro-translocated from the ER to the cytosol where they are degraded by the proteasome (see Kaufman, this Perspective series, ref. 42). In other cases, the oxidizing nature of the ER compartment contributes to the observation of disulfide-cross-linked aggregates within the compartment; correspondingly, a critical role in protein folding and unfolding has been recognized not only for classical chaperones like BiP, but also for oxidoreductases and for a lectin/glucosyltransferase system (see, for instance, refs. 43-45).

\section{Amyloid formation in human disease}

The term "amyloid" was coined in 1853 by Virchow to connote waxy, eosinophilic tissue deposits that he thought were composed of carbohydrate. Within 10 years, however, Friedrich and Kekule established that the deposits were composed of protein. In 1959, with examination by EM, it became clear that there were fibrillar components to such deposits (46). Isolated fibrils were observed to be rigid and nonbranching and to stain characteristically with Congo red dye. EM further revealed that the fibrils were composed of several laterally associated protein filaments, the latter measuring about $75 \AA$ in diameter (47). In 1968 and 1969, two groups $(48,49)$ employed $x$-ray fiber diffraction to show that the spacing of strands within sheets of human amyloid was consistent with a $\beta$-pleated sheet arrangement in which the sheets form a cross- $\beta$ pattern whose spacing is dictated by the projecting side chains.

With improving methods to purify amyloid fibrils, Glenner and coworkers, in 1970, were the first to identify a constituent protein of an amyloid, an Ig light chain in amyloid from patients with primary systemic amyloidosis (50). Glenner's review of the field in 1980 was prescient. He described a "unifying definition of the amyloid fibril as having, in all cases, a $\beta$-pleated sheet structure," and took the view that "amyloid accumulation is not the consequence of a single disease process . .. but a variety of different disease processes ... resulting in the deposition of twisted $\beta$-pleated sheet fibrils formed from various proteins ..." (51). At that point it was already known that amyloid plaques were characteristic of Alzheimer disease and of the spongiform encephalopathies, such as kuru and Creutzfeldt-Jacob disease, and that they were present in the cardiac system of many elderly patients. Today, we know that the deposits in these conditions contain $\mathrm{A} \beta$-peptides, prion protein, and transthyretin (TTR), respectively. Joining this list are such other proteins as islet amyloid protein in the pancreas of patients with type 2 diabetes, lysozyme in the liver of patients with systemic amyloidosis, and cystatin $C$ in patients with hereditary cerebral amyloid angiopathy (52).

Remarkably, the various proteins present in amyloid in these clinical disorders share no primary amino acid sequence, nor even tertiary structural features in their native forms, yet all can assume the same basic cross- $\beta$ sheet topology in amyloid fibrils. Recent results in vitro suggest that any protein can be converted to an amyloid state if exposed to the proper partial-denaturing condition. Even the all- $\alpha$-helical protein apomyoglobin has been incorporated into amyloid fibrils when exposed to $\mathrm{pH} 9$ and $65^{\circ} \mathrm{C}(53)$. Thus, the amyloid state may be accessible to any protein as a very stable alternative conformational state, a state that, despite involving only noncovalent contacts between subunits, does not readily dissociate to monomer, even with exposure to powerful denaturants. Indeed, despite earlier assumptions to the contrary, proteins in amyloids may occupy a state that is even more thermodynamically stable than the native state.

Surely, this nonfunctional dead-end state is one to be avoided in vivo, and, as Dobson and others have commented, evolution has likely selected against residues or sequences within extant proteins that favor conversion to an amyloid conformation (54). Thus, with protein sequences as they have evolved, very high kinetic barriers keep most of our proteins out of this alternative energetic minimum. Why are amyloid structures so stable, why are apparently all proteins accessible to these states, and how are amyloid states accessed?

Amyloid structure. While we still lack a high-resolution structure for any protein in its amyloid form, recent fiber diffraction and EM studies have provided further insight into the cross- $\beta$-sheet structures found in amyloid fibrils. Blake and Serpell used synchrotron radiation in fiber diffraction of TTR amyloids to derive a model of the amyloid fibril consisting of a helical staircase of $\beta$-sheets in which four $\beta$-sheets form the cross section of a protofilament. The individual units in the model comprise structurally rearranged TTR monomers or dimers (55). A cryoEM reconstruction study by Helen Saibil and coworkers of an amyloid formed from an SH3 domain also concluded that the protein must undergo structural rearrangement relative to its native fold (56) (Figure 6). Here, the fibril was found to be composed of four protofilaments, smaller than those of the TTR amyloid and consisting of two stacked $\beta$-sheets (Figure 6, b and c). Crucially, the amyloid structures formed by both TTR and the SH3 domain fail to accommodate the native structure of the protein. 


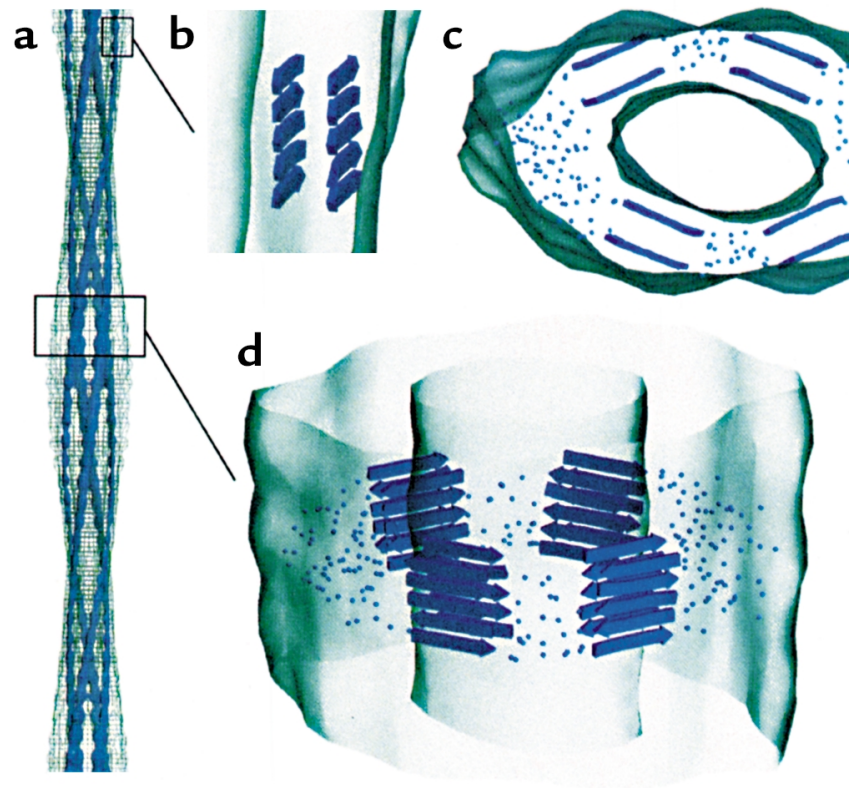

Additional EM studies of a yeast prion protein that can form amyloids in vitro indicate that the entire protein species is not required to become incorporated into the $\beta$-sheet organization of a fibril. In this case, an $\mathrm{NH}_{2}$-terminal region of the protein becomes incorporated into the fibrils themselves, while the remaining $\mathrm{COOH}$-terminal portion of the protein is "marginated" to the outside of the fibril (57). This region is likely to retain a native structure, and there are recent examples of fusion proteins incorporated into amyloids in which a nonamyloidogenic "passenger" enzyme segment retains enzymatic activity after the protein is incorporated into fibrils.

A point that seems to emerge from both the diffraction and the EM studies is that the primary contacts that glue an amyloid protofilament together along its length are the hydrogen bonds between $\beta$-strands. Since these are main-chain contacts, there may be minimal side-chain involvement and therefore little or no sequence specificity to such behavior. Thus, it may be that any segment of polypeptide that can be folded or refolded into $\beta$-strand can ultimately participate in such a stable structure.

Production of amyloidogenic folding intermediates by destabilization of the native state. Evidence has emerged over the past several years to indicate that production of the amyloid state of a protein often involves destabilization of the already-folded native protein. That is, disruption of the globular structure, where a sequence of side chains has dictated a native fold, can dispose to refolding into a structure where main-chain contacts, rather than side-chain interactions, predominate.

It is important at this point to be clear about the definitions of stability and destabilization. These terms are often used in a general structural sense to refer to the effects of mutations or conditions, such as low $\mathrm{pH}$ or the presence of denaturants, that disrupt the native conformation of a protein. It is desirable, however, to distinguish whether such a structural disruption

\section{Figure 6}

Model of an $\mathrm{SH} 3$ amyloid fibril as deduced from cryoEM microscopy of Saibil and coworkers (56). (a) Fibril structure showing four protofilaments and a fairly hollow interior space. (b) Side view of a single protofilament. (c and $\mathbf{d}$ ) Model for packing of $\beta$-sheets into the fibril. Dots indicate regions of polypeptide connecting the $\beta$-sheet regions. Reproduced with permission from The EMBO Journal (56).

reflects an effect on thermodynamic or kinetic stability. The former refers to the free energy difference between the native state and a monomeric intermediate on the pathway to amyloidogenesis and is reflected in the equilibrium between the native and amyloidogenic intermediate states. Mutations generally decrease the thermodynamic stability of a protein by increasing the free energy of the native state, although decreasing the free energy of the intermediate state is also possible. Kinetic stability refers to the height of the free energy barrier between the native state and the transition state for producing the amyloidogenic intermediate and is reflected in the rate constant for conversion from native to intermediate. Mutations affect this rate either by increasing the free energy of the native state or by decreasing that of the transition state. (Note that the relative free energy of the intermediate does not contribute to this effect.)

In many biological systems, both thermodynamic and kinetic effects occur simultaneously with mutation or changing conditions. However, as mentioned above, the kinetic barriers between native and amyloidogenic intermediate states are probably very large in the normal cell, effectively precluding the establishment of an equilibrium between these states on biological time scales. In vitro, however, such a kinetic barrier can be surmounted by partial denaturation, as occurs when the $\mathrm{pH}$ is reduced to $3-4.5$ or in the presence of moderate concentrations of alcohols.

Amyloid formation from mutant proteins. As with the inclusion body-forming aggregates, it is the formation of a non-native monomer intermediate that appears to be critical to the aggregation step in amyloid formation. Here, however, instead of forming from an unfolded state, the intermediate is typically formed from the already-folded native protein. While intermediates of both inclusion body and amyloid reactions have nativelike properties, amyloid formation appears to also require the reformation or reorganization of some elements of secondary and tertiary structure that disposes to association of these intermediates into amyloid fibrils. Both in vitro and in vivo, this process can be potentiated by mutations that structurally destabilize the native state, as demonstrated particularly dramatically for monomeric lysozyme and tetrameric TTR (but see also ref. 58 concerning Ig light chain).

In the case of lysozyme, a $14-\mathrm{kDa}$ secretory protein composed of an $\alpha$-helical and a $\beta$-sheet domain, two 
known human mutations predisposing to amyloidforming disease have been studied in detail (59). Crystal structures of the two mutant proteins in their native forms show them to resemble the wild-type version, although subtle changes suggest that the domain interface might be destabilized. Indeed, the activity of the variant proteins in vitro is more sensitive to heat treatment, which leads to the formation of fibrils with a predominantly $\beta$ structure. Interestingly, fibrils obtained directly from patients, who are typically heterozygous for these (dominant) mutations, contain only the mutant lysozyme, indicating that only the mutant protein populates an amyloidogenic intermediate. Most astonishing in this regard, when hydrogen-deuterium exchange is carried out at physiologic temperature, the mutant proteins prove to be fully exchangeable on the time scale of minutes, whereas the wild-type protein contains 55 nonexchangeable protons. Thus, under conditions where the wild-type native protein is stable, the mutant proteins are very unstable, apparently populating aggregation-prone, partly folded, amyloidogenic states (Figure $7 \mathrm{~b}$ ). Based on the earlier classic hen lysozyme folding studies of Dobson's group (59), where the $\alpha$-domain became structured in intermediate states while the $\beta$-domain was still unstructured, it has been suggested that fibrillogenesis occurs through the $\beta$-domain of the unstable monomer (Figure 7, b and d).

In the case of TTR, destabilization of the tetramer's quaternary structure leads to amyloid formation in vivo, either in the setting of dominant inherited mutations in familial amyloid polyneuropathy or, sporadically, in senile cardiac amyloidosis; in vitro acid treatment ( $\mathrm{pH} 3.5-4.0)$ has similar effects. The rate-limiting step appears to be tetramer dissociation, which is followed by a structural rearrangement of the product monomer to produce an amyloidogenic species that assembles into fibrils (summarized in ref. 60). Circular dichroism studies at $\mathrm{pH} 3.6$ show that the amyloidogenic conformation retains a secondary-structure content resembling that of the native protein (61). Consistent with a model requiring tetramer dissociation, a homotetramer of the most pathogenic TTR mutant, L55P, has been found to be much less stable than wildtype to acid treatment in vitro, dissociating and forming fibrils under mildly acidic conditions that do not affect wild-type TTR (62). Another prevalent amyloidogenic mutant, V30M, is also sensitive to modest drops in $\mathrm{pH}$ but can be stabilized by the presence of a second mutation, T119M, either within the same subunit (62) or in trans, as one or more separate subunits in the same tetramer (63). This latter configuration corresponds to the milder clinical condition observed in patients who are compound heterozygotes for V30M and T119M. Consistent with this, T119M tetramers are far more stable in vitro against acid disruption than are wild-type tetramers. Finally, a recent study in which a monomeric variant of TTR was engineered by mutating residues in the dimerization interfaces (64) showed that the protein, which is stable and nonamyloidogenic at $\mathrm{pH} 7$, undergoes a rapid conformational rearrangement and generates fibrils at mildly acidic $\mathrm{pH}$. Here, with tetramer dissociation no longer a requirement, presumably the only limiting step to fibril formation is tertiary structural rearrangement.

Efforts to better characterize the TTR amyloidogenic intermediate include tryptophan fluorescence studies suggesting that edge strands of one of the $\beta$-sheets become conformationally altered, and binding studies with the hydrophobic-binding dye ANS (8-anilino-naphthalene-sulfonic acid), which suggest that this intermediate does not expose significant hydrophobic surface (60). A deuterium-hydrogen-exchange nuclear magnetic resonance study has shown that the interstrand hydrogen bonds of one of the sheets of TTR are disrupted at $\mathrm{pH} 4.5$, indicating that sheet destabilization contributes to the amyloidogenic conformation (65).

Consistent with a role for kinetic control of TTR amyloid formation, the mutation V122I has recently been shown to increase the rate of TTR tetramer dissociation under certain conditions (66). A case for kinetic control has also been made for the prion protein, where many of the inherited mutations disposing to disease have been found to have no effect on thermodynamic stability of $\mathrm{PrP}$, as measured by urea denaturation curves following circular dichroism spectra $(67,68)$. Similar analyses of mouse PrP show that the amyloid form is thermodynamically more stable than the native $\alpha$-helical form but is separated from it by a high kinetic barrier (69).

Thermodynamic control of polymerization by amyloidogenic intermediates. Amyloidogenic intermediates must polymerize to form fibrils. As with many other polymerization reactions (microtubule growth, flagellum

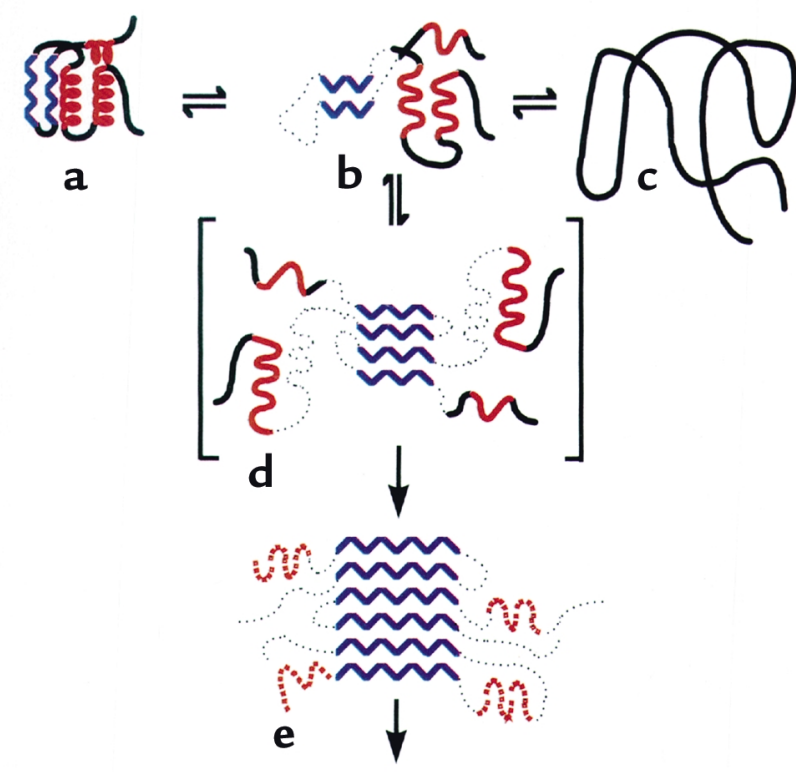

Further assembly of protofilaments

Figure 7

Mechanism proposed for lysozyme amyloidosis (59). An amyloidogenic intermediate state $(\mathbf{b})$ formed from destabilization of the native structure (a) is proposed to self-associate through $\beta$-strand elements (blue) to initiate fibril formation (d, e), rather than forming non-native monomer (c). Reproduced with permission from Nature (59). 

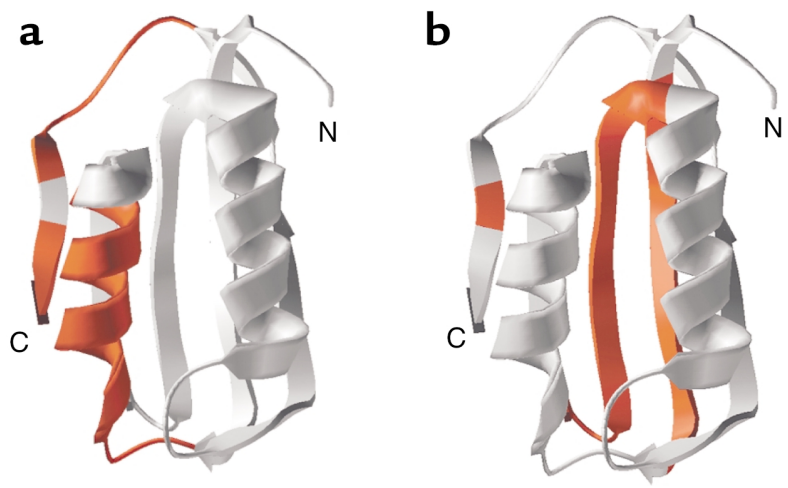

\section{Figure 8}

Distinct regions of acylphosphatase govern alternative fates of aggregation to form amyloid (a) or productive folding to native form $(\mathbf{b}) . \mathrm{N}, \mathrm{N}$ terminus; C, C-terminus of the polypepetide chain. Reproduced with permission from Nature Structural Biology (73).

formation, and viral coat assembly, for instance), nucleation may be a crucial step in this process for at least some amyloid species (70). An oligomeric assembly of a nucleus of a critical size can form from amyloidogenic monomers by a series of association reactions that have, overall, an unfavorable equilibrium. Hence, nucleus formation is under thermodynamic control. Once formed, however, the nucleus can rapidly polymerize into an amyloid fibril.

This behavior predicts that there will be a critical concentration of monomers below which amyloid cannot form; that, once the threshold concentration is reached, there will be a lag time before the association reactions permit production of a nucleus; and that addition of a preformed nucleus during the lag can result in immediate production of amyloid fibrils. While these predictions have been met in vitro for $\mathrm{PrP}$, for $A \beta$-peptide, and for several other amyloid precursors, this mechanism remains to be established in vivo. For example, only prion diseases show the phenomenon of seeding, where inoculation of amyloid material produces disease. Nevertheless, nucleation-polymerization behavior might account, at least in part, for the very long development times for amyloid disease, even in the setting of mutations that destabilize the native state of an amyloidogenic protein.

At a further level of complexity, two recent studies indicate that nonfibrillar, in one case amorphous, aggregates of amyloidogenic proteins, formed early on a fibrillogenesis pathway, may be toxic to cells or brain function $(71$, 72). By contrast, mature fibrils do not exhibit such toxicity. Whether these amorphous structures are incipient fibrils or are structurally disordered states from which ordered aggregates are formed remains unknown.

Domain structures that influence amyloidogenesis. Recent studies in vitro of the two-state folding protein acylphosphatase suggest that different regions of this protein are responsible for favoring pathways of folding to native form versus those leading to amyloid (73) (Figure 8). In particular, mutations in two regions of the protein, amino acids $16-31$, mapping into $\alpha$-helix 1 , and amino acids 87-98, mapping into a terminal $\beta$-strand, interfere with amyloid formation promoted by denaturation in trifluoroethanol (Figure 8a). These regions exhibit significant hydrophobicity and $\beta$-sheet-forming propensity, despite the fact that amino acids $16-31$ form an $\alpha$-helix in the native state. By contrast, a distinct region, exhibiting structure even in the transition state, has been found to be critical for productive folding in aqueous solvent (Figure $8 \mathrm{~b}$ ). Thus, properties of different regions of the same chain can dictate which intermediates form and thereby influence the protein's final fate, albeit that under physiologic conditions, evolution seems to have chosen that de novo folding originating from the ribosome should favor the pathway to the native state.

Prospects for clinical control of amyloid diseases. The foregoing considerations have important implications for the formation of amyloids in the clinical setting. First, thankfully, it appears that only a limited number of proteins have access to an amyloid state in vivo. Almost all of those that can form amyloid are secreted proteins like lysozyme, TTR, and PrP. At this point, it is unclear whether these proteins undergo conformational conversion in the intracellular environment, perhaps in the mildly acidic compartment of the endosome, or whether conversion occurs outside the cell. Regardless, it is clear that chaperone systems that can normally forestall aggregation events fail to prevent these proteins from undergoing the transition to form amyloids. It may be simply a compartmentation problem: If amyloid formation occurs in the endosomes or outside of the cell, chaperones are absent and there is no safeguard system available. Alternatively, if the intermediates formed are sufficiently structured, failing to expose significant hydrophobicity, as appears to be the case with TTR, the chaperone system might not recognize these species. Perhaps this is the case for other amyloids, formed in compartments that are well-populated by molecular chaperones. For example, polyglutamine repeat expansion disorders involve proteins that are cytosolic or nuclear (74). While chaperones as well as proteasome components have been found associated with polyglutamine aggregates, their role in aggregation remains unclear. Likewise, $\alpha$-synuclein aggregation in Parkinson disease occurs in the cytosol, and occasional aggregates observed in postmortem tissue stain positively for Hsp70 or Hsp40 chaperones. Finally, recent studies have suggested that aggregates themselves may inhibit the normal proteasome system that helps clear misfolded or aggregation-prone proteins (75).

Among the various amyloidogenic proteins, $\operatorname{PrP}$ is unique with regard to its infectious behavior. The notion that the prion form, $\mathrm{PrP}^{\mathrm{Sc}}$, can produce conformational conversion of the wild-type $\operatorname{Pr}^{c}$ protein predicts a behavior that is not generally observed for the other amyloidogenic species (76). Whether this relates somehow to the pathway of clinical infection or to the conformational states of the protein remains to be addressed.

If structural destabilization of the native state is the major contributor to amyloidogenesis in these various clinical conditions, then, as proposed by Kelly and colleagues, stabilizing the native form of a protein by 
binding a ligand may offer one of the most direct means of preventing disease (77). In the case of TTR, for example, providing a compound that mimics the normal ligand, thyroxine, and stabilizes the tetramer against dissociation is an approach currently being tested (78). By contrast, disease produced by A $\beta$-peptides will not be easily addressable in this manner, as these peptides are proteolytic products that have no favored conformational state other than the amyloidogenic ones (5). Obviously, stabilizing intact APP itself against $\beta$ or $\gamma$ proteolysis, or developing inhibitors of the proteases, may help prevent such amyloid formation. For prion disease a number of compounds are being tested, including quinacrine and chlorpromazine, that block PrPSc production and may also enhance its clearance (79). In sum, the particular conformational pathway taken by each of the clinically relevant amyloidogenic proteins provides, in each case where a pathway becomes understood, a specific avenue for development of compounds that block partitioning of that protein to amyloid.

\section{Acknowledgments}

I thank Wayne Fenton for discussion and critical reading of the manuscript, and Howard Hughes Medical Institute for its generous support.

1. Weatherall, D.J., Clegg, J.B., Higgs, D.R., and Wood, W.G. 2001. The hemoglobinopathies. In Metabolic and molecular bases of inherited disease. C. Scriver, A. Beaudet, D. Valle, and W. Sly, editors. McGraw-Hill. New York, New York, USA. 4571-4636.

2. Perlmutter, D.H. 2002. Liver injury in $\alpha_{1}$-antitrypsin deficiency: role of autophagy and mitochondrial injury in response to aggregated protein in the endoplasmic reticulum. J. Clin. Invest. In press.

3. Cox, D.W. 2001. $\alpha_{1}$-Antitrypsin deficiency. In Metabolic and molecular bases of inherited disease. C. Scriver, A. Beaudet, D. Valle, and W. Sly, editors. McGraw-Hill. New York, New York, USA. 5559-5584.

4. Selkoe, D.J. 2002. Deciphering the genesis and fate of amyloid- $\beta$ protein yields novel therapies for Alzheimer disease. J. Clin. Invest. In press.

5. Selkoe, D. 1997. Cellular and molecular biology of the beta-amyloid precursor protein and Alzheimer's disease. In The molecular and genetic basis of neurological disease. R.N. Rosenberg, S.B. Prusiner, S. DiMauro, and R.L. Barchi, editors. Butterworth-Heinemann. Boston, Massachusetts, USA. 601-611.

6. DeArmond, S.J., and Prusiner, S.B. 1997. Molecular neuropathology of prion diseases. In The molecular and genetic basis of neurological disease. R.N. Rosenberg, S.B. Prusiner, S. DiMauro, and R.L. Barchi, editors. Butterworth-Heinemann. Boston, Massachusetts, USA. 145-163.

7. DiFiglia, M., et al. 1997. Aggregation of huntingtin in neuronal intranuclear inclusions and dystrophic neurites in brain. Science. 277:1990-1993.

8. Scherzinger, E., et al. 1997. Huntintin-encoded polyglutamine expansions form amyloid-like protein aggregates in vitro and in vivo. Cell. 90:549-558.

9. Conway, K.A., Harper, J.D., and Lansbury, P.T. 2000. Fibrils formed in vitro from $\alpha$-synuclein and two mutant forms linked to Parkinson's disease are typical amyloid. Biochemistry. 39:2552-2563.

10. Serpell, L.C., Berriman, J., Jakes, R., Goedert, M., and Crowther, R.A. 2000 . Fiber diffraction of synthetic $\alpha$-synuclein filaments shows amyloid-like cross- $\beta$ conformation. Proc. Natl. Acad. Sci. USA. 97:4897-4902.

11. Anfinsen, C.B. 1973. Principles that govern the folding of protein chains. Science. 181:223-230.

12. Fersht, A.R. 1995. Characterizing transition states in protein folding: an essential step in the puzzle. Curr. Opin. Struct. Biol. 5:79-84.

13. Baker, D. 2000. A surprising simplicity to protein folding. Nature. 405:39-42.

14. Kim, P.S., and Baldwin, R.L. 1990. Intermediates in the folding reactions of small proteins. Annu. Rev. Biochem. 59:631-660.

15. Silow, M., Tan, Y.-J., Fersht, A.R., and Oliveberg, M. 1999. Formation of short-lived protein aggregates directly from the coil in two-state folding. Biochemistry. 38:13006-13012.

16. London, J., Skrzynia, C., and Goldberg, M.E. 1974. Renaturation of Escherichia coli tryptophanase after exposure to $8 \mathrm{M}$ urea. Eur. J. Biochem. 47:409-415.
17. Zettlmeissl, G., Rudolph, R., and Jaenicke, R. 1979. Reconstitution of lactic dehydrogenase. Noncovalent aggregation vs. reactivation. Biochemistry. 18:5567-5571.

18. Mitraki, A., Betton, J.-M., Desmadril, M., and Yon, J.M. 1987. Quasi-irreversibility in the unfolding-refolding transition of phosphoglycerate kinase induced by guanidine hydrochloride. Eur. J. Biochem. 163:29-34.

19. Brems, D.N. 1988. Solubility of different folding conformers of bovine growth hormone. Biochemistry. 27:4541-4546.

20. Steinbacher, S., et al. 1994. Crystal structure of P22 tailspike protein: interdigitated subunits in a thermostable trimer. Science. 265:383-386.

21. Haase-Pettingell, C.A., and King, J. 1988. Formation of aggregates from a thermolabile in vivo folding intermediate in P22 tailspike maturation. J. Biol. Chem. 263:4977-4983.

22. King, J., Haase-Pettingell, C., Robinson, A.S., Speed, M., and Mitraki, A. 1996. Thermolabile folding intermediates: inclusion body precursors and chaperonin substrates. FASEB J. 10:57-66.

23. Mitraki, A., Fane, B., Haase-Pettingell, C., Sturtevant, J., and King, J. 1991. Global suppression of protein folding defects and inclusion body formation. Science. 253:54-58.

24. Gordon, C.L., and King, J. 1993. Temperature-sensitive mutations in the phage P22 coat protein which interfere with polypeptide chain folding. J. Biol. Chem. 268:9358-9368.

25. Speed, M.A., Wang, D.I.C., and King, J. 1996. Specific aggregation of partially folded polypeptide chains: the molecular basis of inclusion body composition. Nat. Biotechnol. 14:1283-1287.

26. Bennett, M.J., Choe, S., and Eisenberg, D. 1994. Domain swapping: entangling alliances between proteins. Proc. Natl. Acad. Sci. USA. 91:3127-3131.

27. Bennett, M.J., Schlunegger, M.P., and Eisenberg, D. 1995. 3D domain swapping: a mechanism for oligomer assembly. Protein Sci. 4:2455-2468.

28. Lomas, D., and Mahadeva, R. 2002. $\alpha_{1}$-Antitrypsin polymerization and the serpinopath: pathobiology and prospects for therapy. J. Clin. Invest. In press.

29. Lomas, D.A., Evans, D.L., Finch, J.T., and Carrell, R.W. 1992. The mechanism of $Z \alpha_{1}$-antitrypsin accumulation in the liver. Nature. 357:605-607.

30. Pelham, H.R. 1986. Speculations on the functions of the major heat shock and glucose-regulated proteins. Cell. 46:959-961.

31. Bukau, B., and Horwich, A.L. 1998. The Hsp70 and Hsp60 chaperone machines. Cell. 92:351-366.

32. Cheng, M.Y., et al. 1989. Mitochondrial heat-shock protein Hsp60 is essential for assembly of proteins imported into yeast mitochondria. Nature. 337:620-625.

33. Goloubinoff, P., Christeller, J.T., Gatenby, A.A., and Lorimer, G.H. 1989. Reconstitution of active dimeric ribulose bisphosphate carboxylase from an unfolded state depends on two chaperonin proteins and MgATP. Nature. 342:884-889.

34. Martin, J., et al. 1991. Chaperonin-mediated protein folding at the surface of groEL through a "molten globule"-like intermediate. Nature. 352:36-42.

35. Ranson, N.A., Dunster, N.J., Burston, S.G., and Clarke, A.R. 1995. Chaperonins can catalyze the reversal of early aggregations steps when a protein misfolds. J. Mol. Biol. 250:581-586.

36. Braig, K., et al. 1994. The crystal structure of the bacterial chaperonin GroEL at 2.8 A. Nature. 371:578-586.

37. Grantcharova, V., Alm, E.J., Baker, D., and Horwich, A.L. 2001. Mechanisms of protein folding. Curr. Opin. Struct. Biol. 11:70-82.

38. Xu, Z., Horwich, A.L., and Sigler, P.B. 1997. The crystal structure of the asymmetric GroEL-GroES-(ADP) 7 chaperonin complex. Nature. 388:741-750.

39. Gordon, C.L., Sather, S.K., Casjens, S., and King, J. 1994. Selective in vivo rescue by GroEL-ES of thermolabile folding intermediate to phage P22 structural proteins. J. Biol. Chem. 269:27941-27951.

40. Glover, J.R., and Lindquist, S. 1998. Hsp104, Hsp70, and Hsp40: a novel chaperone system that rescues previously aggregated proteins. Cell. 94:73-82.

41. Parsell, D.A., Kowal, A.S., Singer, M.A., and Lindquist, S. 1994. Protein disaggregation mediated by heat-shock protein Hsp104. Nature. 372:475-478.

42. Kaufman, R. 2002. Orchestrating the unfolded protein response in health and disease. J. Clin. Invest. In press.

43. Tu, B.P., Ho-Schleyer, S.C., Travers, K.J., and Weissman, J.S. 2000. Biochemical basis of oxidative protein folding in the endoplasmic reticulum. Science. 290:1571-1574.

44. Tsai, B., Rodighiero, C., Lencer, W.I., and Rapoport, T.A. 2001. Protein disulfide isomerase acts as a redox-dependent chaperone to unfold cholera toxin. Cell. 104:937-948.

45. Helenius, A., and Aebi, M. 2001. Intracellular functions of N-linked glycans. Science. 291:2364-2369.

46. Cohen, A.S., and Calkins, E. 1959. Electron microscopic observation on a fibrous component in amyloid of diverse origins. Nature. 183:1202-1203.

47. Shirahama, T., and Cohen, A.S. 1967. High-resolution electron micro- 
scopic analysis of the amyloid fibril. J. Cell Biol. 33:679-708.

48. Eanes, E.D., and Glenner, G.G. 1968. X-ray diffraction studies on amyloid filaments. J. Histochem. Cytochem. 16:673-677.

49. Bonar, L., Cohen, A.S., and Skinner, M.M. 1969. Characterization of the amyloid fibril as a cross- $\beta$ protein. Proc. Soc. Exp. Biol. Med. 131:1373-1375.

50. Glenner, G.G., Harbaugh, J., Ohms, J.I., Harada, M., and Cuatrecasas, P. 1970. An amyloid protein: the amino-terminal variable fragment of an immunoglobulin light chain. Biochem. Biophys. Res. Commun. 41:1287-1289.

51. Glenner, G.G. 1980. Amyloid deposits and amyloidosis: the $\beta$-fibrilloses. N. Engl. J. Med. 302:1283-1292.

52. Kelly, J.W. 1996. Alternative conformations of amyloidogenic proteins govern their behaviour. Curr. Opin. Struct. Biol. 6:11-17.

53. Fändrich, M., Fletcher, M.A., and Dobson, C.M. 2001. Amyloid fibrils from muscle myloglobin. Nature. 410:165-166.

54. Dobson, C.M. 1999. Protein misfolding, evolution and disease. Trends Biochem. Sci. 24:329-332.

55. Blake, C., and Serpell, L. 1996. Synchrotron X-ray studies suggest that the core of the transthyretin amyloid fibril is a continuous $\beta$-sheet helix. Structure. 4:989-998.

56. Jimenez, J.L., et al. 1999. Cryo-electron microscopy structure of an SH3 amyloid fibril and model of the molecular packing. EMBOJ. 18:815-821.

57. Glover, J.R., et al. 1997. Self-seeded fibers formed by Sup35, the protein determinant of $\left[\mathrm{PSI}^{+}\right]$, a heritable prion-like factor of S.cerevisiae. Cell. 89:811-819.

58. Hurle, M.R., Helms, L.R., Li, L., Chan, W., and Wetzel, R. 1994. A role for destabilizing amino acid replacements in light-chain amyloidosis. Proc. Natl. Acad. Sci. USA. 91:5446-5450.

59. Booth, D.R., et al. 1997. Instability, unfolding and aggregation of human lysozyme variants underlying amyloid fibrillogenesis. Nature. 385:787-793.

60. Lai, Z., Colon, W., and Kelly, J.W. 1996. The acid-mediated denaturation pathway of transthyretin yields a conformational intermediate that can self-assemble into amyloid. Biochemistry. 35:6470-6482.

61. Colon, W., and Kelly, J.W. 1992. Partial denaturation of transthyretin is sufficient for amyloid fibril formation in vitro. Biochemistry. 31:8654-8660.

62. McCutchen, S.L., Lai, Z., Miroy, G.J., Kelly, J.W., and Colon, W. 1995. Comparison of lethal and nonlethal transthyretin variants and their relationship to amyloid disease. Biochemistry. 34:13527-13536.

63. Hammarström, P., Schneider, F., and Kelly, J.W. 2001. Trans-suppression of misfolding in an amyloid disease. Science. 293:2459-2462.

64. Jiang, X., et al. 2001. An engineered transthyretin monomer that is nonamyloidogenic, unless it is partially denatured. Biochemistry. 40:11442-11452.
65. Liu, K., Cho, H.S., Lashuel, H.A., Kelly, J.W., and Wemmer, D.E. 2000. A glimpse of a possible amyloidogenic intermediate of transthyretin. Nat. Struct. Biol. 7:754-757.

66. Jiang, X., Buxbaum, J.N., and Kelly, J.W. 2001. The V122I cardiomyophathy variant of transthyretin increases the velocity of rate-limiting tetramer dissociation, resulting in accelerated amyloidosis. Proc. Natl. Acad. Sci. USA. 98:14943-14948.

67. Swietnicki, W., Petersen, R.B., Gambetti, P., and Surewicz, W.K. 1998. Familial mutations and the thermodynamic stability of the recombinant human prion protein. J. Biol. Chem. 273:31048-31052.

68. Liemann, S., and Glockshuber, R. 1999. Influence of amino acid substitutions related to inherited human prion diseases on the thermodynamic stability of the cellular prion protein. Biochemistry. 38:3258-3267.

69. Baskakov, I.V., Legname, G., Prusiner, S.B., and Cohen, F.E. 2001. Folding of prion protein to its native alpha-helical conformation is under kinetic control. J. Biol. Chem. 276:19687-19690.

70. Harper, J.D., and Lansbury, P.T. 1997. Models of amyloid seeding in Alzheimer's disease and scrapie. Annu. Rev. Biochem. 66:385-407.

71. Bucciantini, M., Giannoni, E., Chiti, F., Baroni, F., and Formigli, L. 2002. Inherent toxicity of aggregates implies a common mechanism for protein misfolding diseases. Nature. 416:507-511.

72. Walsh, D.M., et al. 2002. Naturally secreted oligomers of amyloid $\beta$ protein potently inhibit hippocampal long-term potentiation in vivo. Nature. 416:535-539.

73. Chiti, F., et al. 2002. Kinetic partitioning of protein folding and aggregation. Nat. Struct. Biol. 9:137-143.

74. Perutz, M.F., Johnson, T., Suzuki, M., and Finch, J.T. 1994. Glutamine repeats as polar zippers: their possible role in inherited neurodegenerative diseases. Proc. Natl. Acad. Sci. USA. 91:5355-5358.

75. Bence, N.F., Sampat, R.M., and Kopito, R.R. 2001. Impairment of the ubiquitin-proteasome system by protein aggregation. Science. 292:1552-1555.

76. Prusiner, S.B. 2001. Shattuck lecture: neurodegenerative diseases and prions. N. Engl. J. Med. 244:1516-1526.

77. Miroy, G.J., et al. 1996. Inhibiting transthyretin amyloid fibril formation via protein stabilization. Proc. Natl. Acad. Sci. USA. 93:15051-15056.

78. Klabunde, T., et al. 2000. Rational design of potent human transthyretin amyloid disease inhibitors. Nat. Struct. Biol. 7:312-321.

79. Korth, C., May, B.C.H., Cohen, F.E., and Prusiner, S.B. 2001. Acridine and phenothiazine derivatives as pharmacotherapeutics for prion disease. Proc. Natl. Acad. Sci. USA. 98:9836-9841.

80. Dill, K.A., and Chan, H.S. 1997. From Levinthal to pathways to funnels. Nat. Struct. Biol. 4:10-19. 